East African Medical Journal Vol. 77 No. 6 June 2000

HYDRANENCEPHALY IN MALAWIAN CHILDREN

A. Adeloye, FRCS, FRCP, Neurosurgery Unit, Department of Surgery, College of Medicine, University of Malawi, Private Bag 360, Chichiri, Blantyre, Malawi

\title{
HYDRANENCEPHALY IN MALAWIAN CHILDREN
}

\section{A. ADELOYE}

\begin{abstract}
Objective: To show that not all big heads in children are due to hydrocephalus.

Setting: The Queen Elizabeth Central Hospital (QECH), Blantyre, the teaching hospital of Malawi College of Medicine. It is the biggest and leading hospital in Malawi and the major referral centre for the whole country.

Design: A prospective study was made of all children who presented at QECH, Blantyre with the diagnosis of hydrocephalus during the study period.

Subjects: Fifteen cases of hydranencephaly were encountered.

Intervention: All cases of abnormally large heads were investigated with cranial ultrasonography, a few children had air ventriculography and CT scan of the brain. All patients had ventricular aspiration of cerebral spinal fluid (CSF) to verify the colour and condition of the fluid before performing ventriculo-peritoneal shunting for which our locally made Malawi unishunt was used.

Results: In hydranencephaly, the brain was found replaced by large fluid-filled cavity in contrast to hydrocephalus where brain was present and ventricular dilatation was encountered. Ventriculo-peritoneal shunting benefited children with hydrocephalus but not those with hydranencephaly.

Conclusion: Hydranencephaly is one of the causes of abnormal head enlargement in children often confused with hydrocephalus. It is important to recognise hydranencephaly to avoid unnecessary operation which does not help the patient.
\end{abstract}

\section{INTRODUCTION}

Hydranencephaly is a cause of abnormal head enlargement in a child which can easily be mistaken for hydrocephalus. Pathologically in hydranencephaly, the cerebral hemispheres are absent or significantly reduced and represented by a sac of membranes over which remnants of the frontal, temporal and occipital lobes are scattered. The basal ganglia and the brain stem are present. The observation that the parts of the brain missing in hydranencephaly are fed by the internal carotid artery raised the suggestion that the disease results from bilateral occlusion of the internal carotid artery during foetal life with preservation of the vertebro-basilar circulation $(1,2)$. This intrauterine destructive lesion of the cerebral hemispheres may also be caused by intraventricular haemorrhage, abdominal trauma to the mother, infections such as toxoplasmosis, bacterial meningitis, viral encephalitis and rubella $(3,4)$ and invasion of the brain by congenital neoplastic disease(5).

Ventriculo-peritoneal shunting is good treatment for hydrocephalus but not for hydranencephaly. The purpose of this paper is to draw attention to the clinical, radiological and other features of hydranencephaly for its better recognition in the hope that it will assist the clinician in the selection of those children with abnormally large heads who are more likely than others to profit from the operative procedure of ventriculoperitoneal shunting.

\section{MATERIALS AND METHODS}

During a prospective study of hydrocephalus in Malawi children(6), fifteen cases of hydranencephaly which were encountered between 1992 and 1997 formed the subjects of this report. The children presented with abnormally big heads and were presumed to be suffering from hydrocephalus. The findings on clinical examination, during investigation for hydrocephalus; operative findings and events during their post operative follow up were documented. All the children were investigated with ultrasonography of the head and ventricular aspiration through the right lateral angle of the anterior fontanelle to find out the colour of the CSF. Only one child had CT scan of the brain.

\section{RESULTS}

Clinical findings: Gender and age distribution: There were eight males and seven females aged, at presentation, between two weeks and nine months.

Onset of big head: This was present at birth in one child, among the rest, the large head was noted in $50 \%$ of cases within six months of life and the rest from between six and nine months.

Symptoms and signs: These were similar to those associated with infantile hydrocephalus, excessive crying was a common complaint by the mothers. On physical examination, apart from the common features associated with hydrocephalus, the nutritional state was very good in all cases. The head transilluminated easily. 
The features associated with hydranencephaly in this series include blindness from birth (two cases); hypertelorism alone (one case); hypertelorism associated with low set ears and undescended testes (one case) (Table 1). The blindness presented as roving eyes in one child, in the other child, the mother noted that her child was unable to see the breast when being breast-fed.

Table 1

Associated lesions and findings in hydranencephaly

\begin{tabular}{lc}
\hline Finding & No. of cases \\
\hline Blindness from birth & 2 \\
Hypertelorism alone & 1 \\
$\begin{array}{l}\text { Hypertelorism with low set } \\
\text { ears and undescended testicles }\end{array}$ & 1 \\
\hline
\end{tabular}

Ultrasonographic features: The replacement of the brain by a large fluid-filled cavity was variously described as detailed on Table 2. Figure 1 is the cranial ultrasound picture of a child of four months with hydranencephaly showing fluid filling the anterior and middle cranial fossae and most of the supratentorial space. This is different from Figure 2, the ultrasound of a hydrocephalic four months old child with dilatation of both lateral ventricles and the third ventricle due to aqueductal stenosis.

Table 2

Ultrasonographic findings

- Gross hydrocephalus with little cortical mantle

- Whole brain parenchyma replaced by fluid

- Massive hydrocephalus with no brain

- $\quad$ Large ventricles with no brain tissue suggesting cortical atrophy

\section{Figure 1}

Cranial ultrasound of a child with hydranencephaly

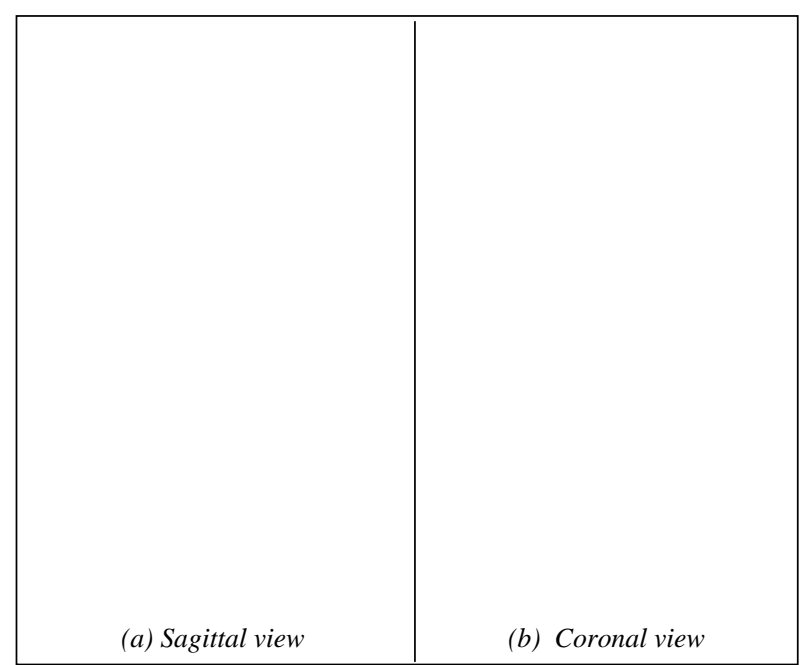

Note fluid filling of the supratentorial space, well formed falx cerebri, brain stem and cerebellum.
Findings on CT scan of the brain: CT scan of the brain was available in one case. It showed macrocephaly with severe hydranencephaly with virtual absence of the cerebral hemispheres with scattered islands of cortex in the occipital and temporal lobes. The thalamus, brainstem and cerebellum were present. The falx cerebri was displaced to the right side so that the left hemicranium was larger than the right.

Figure 2

Cranial ultrasound of a child with obstructive hydrocephalus

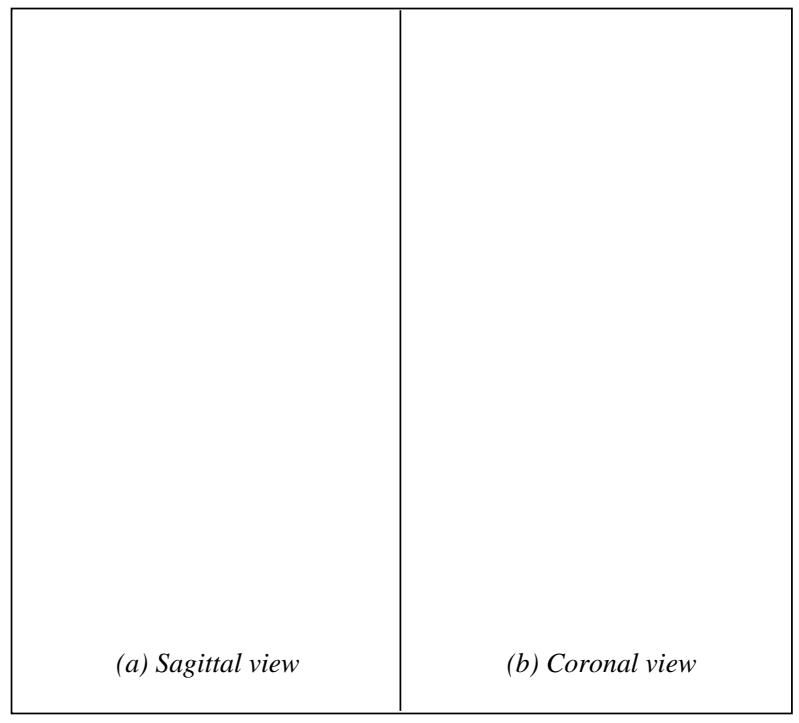

Note dilatation of both lateral ventricles and the third ventricle suggesting aqueductal stenosis

Findings during ventricular aspiration: The CSF obtained from tapping the lateral ventricle through the right lateral angle of the anterior fontanelle was clear and under no elevated pressure in 14 of the 15 children in this series. After removing about 20 to $30 \mathrm{cc}$ of CSF, the anterior fontanelle usually became flat and the head felt very soft.

Only in one case was the CSF haemorrhagic; a repeat ventricular aspiration four weeks later revealed clear CSF, still under no raised pressure.

Operative findings: Six children had venticuloperitoneal ( $\mathrm{v}-\mathrm{p})$ shunting procedure using the low pressure Malawi unishunt(7). These children were presumed to be having hydrocephalus. With subsequent better understanding and diagnosis of hydranencephaly, V-P shunting was not offered other children with hydranencephaly.

(i) Intra-operative observation: At operation, after making the burr hole, the dura was found to be blue in colour. When the dura was opened no brain tissue was encountered; only a thin membrane was found and the CSF was clear. When the ventricular catheter was connected to the lower (abdominal) catheter, the flow of CSF was initially brisk, only to peter out to a slow dribble after releasing some fluid. The anterior fontanelle became flat as soon as the shunt was in place; 
(ii) Post-operative features: The late post-operative features noted included head collapse, delayed milestones and liability to shunt revision or removal due to nonfunctioning of the system.

\section{DISCUSSION}

It is useful for therapeutic purposes to distinguish between normal hydrocephalus and hydranencephaly because the benefit from ventriculo-peritoneal shunt operation is substantial in the child with hydrocephalus when it is not so in case of hydranencephaly.

In both conditions the head size is normal at birth; in both head enlargement begins in the first three months of life(8). Where there is CT scan and or MRI, these imaging techniques help to differentiate hydrocephalus from hydranencephaly by demonstrating in the latter condition no rostal tissue above the midbrain but with retention of the thalamus $(5,9)$. In institutions lacking in modern imaging techniques, we depend on clinical, ultrasonographic and other findings to differentiate between these two diseases.

Clinically in hydranencephaly the abnormal fluid accumulation is contained in the membranous cavity which results in cranial transillumination, in hydrocephalus there is no such membrane and cranial transillumination because there is still some brain tissue to make up the cerebral mantle. On ventricular aspiration, in hydranencephaly the fluid harvested is not under pressure so that it dribbles out slowly leading to flattening of the anterior fontanelle after the removal of little amount of fluid. In hydrocephalus, the CSF is under elevated pressure, substantial amount of it has to be withdrawn to reduce the tension over the anterior fontanelle.

At surgery for ventriculo-peritoneal shunting, as soon as the burr hole is made and the dura matter is exposed, a blue dura is seen in hydranencephaly, in hydrocephalus, the dura is of normal white colour. It is not recommended to go on with the operation since hydranencephaly carries a poor prognosis for psychomotor retardation $(5,10)$.
Hydranencephaly as a cause of head enlargement is often confused with hydrocephalus. The features of hydranencephaly on clinical presentation, ventricular aspiration, ultrasonography and at operation are described for a better recognition of the disease.

\section{REFERENCES}

1. Behrman, R.E. Nelson's Textbook of Pediatrics 14th edition 1992, page 1490 .

2. Halsey, J.H., Allen, N. and Chamberlain, H.R. The morphogenesis of hydranencephaly. J. Neurol. Sci. 1971; 12:187 - 217

3. Deshmukh, C.T., Nadkarni, U.B., Nair, K; Charpure, V.P. and Jain, M.K. Hydranencephaly and multicystic encephalomalacia: association with congenital rubella infection. Indian Paediat. 1993; 30:253.

4. Kubo, S., Kishino, T., Satake, N., Okano, M., Mikawa, M. and Ishikawa, $\mathrm{N}$. A neonatal case of hydranencephaly caused by atheromatous plaque obstruction of aortic arch: possible association with a congenital cytomegalovirus infection? $J$. Perinat. 1994; 14:483 - 6.

5. Velasco, M.E., Brown, J.A., Kini, J. and Ruppert, E.S. Primary congenital rhabdoid tumour of the brain with neoplastic hydranencephaly. Child's Nerv. Syst. 1993; 9:185 -190.

6. Adeloye, A. and Khare, R. Ultrasonographic Study of children suspected of hydrocephalus at the Queen Elizabeth Central Hospital in Blantyre, Malawi. East Afr. Med. J. 1997; 74:56 - 59

7. Adeloye, A. Experience with the use of the Malawi shunt in the treatment of obstructive hydrocephalus in children. East Afr. Med. J. 1997; 74:52 - 55

8. Friede, R L. Porencephaly, hydranencephaly, multilocular cystic encephalopathy Developmental Neurology, 1975 Springer, Wien, pp $102-122$

9. Tayama, M., Hashimoto T. and Mori K., et al. Electrophysiological study on hydranencephaly. Brain and Development. 1992; 14: $185-7$.

10. van Doornik, M.C. and Hernekam, R.C. Herni-hydranencephaly with favourable outcome. Develop. Med. Child Neur. 1992; 34:454 - 8 . 